University of East London Institutional Repository: http://roar.uel.ac.uk

This paper is made available online in accordance with publisher policies. Please scroll down to view the document itself. Please refer to the repository record for this item and our policy information available from the repository home page for further information.

To see the final version of this paper please visit the publisher's website.

Access to the published version may require a subscription.

Author(s): Lota, Jaswinder. Al-Janabi, Mohammed., Kale, Izzet

Article Title: Stability analysis of higher-order delta-sigma modulators using the describing function method

Year of publication: 2006

Citation: Lota, J. Al-Janabi, M., Kale, I. (2006) 'Stability analysis of higher-order delta-sigma modulators using the describing function method' In: Proceedings of the IEEE International Symposium on Circuits and Systems, 2006: ISCAS 2006. IEEE, Los Alamitos, USA, pp. 593-596.

Link to published version: http://dx.doi.org/10.1109/ISCAS.2006.1692655

DOI: $10.1109 /$ ISCAS.2006.1692655

Publisher statement:

http://www.ieee.org/web/publications/rights/policies.html 


\title{
Stability Analysis of Higher-Order Delta-Sigma Modulators using the Describing Function Method
}

\author{
Jaswinder Lota*, MIEEE, Mohammed Al-Janabi*, MIEEE, Izzet Kale* ${ }^{+}$, MIEEE \\ *Applied DSP and VLSI Research Group, Department of Electronic Systems, University of Westminster, London, United Kingdom \\ ${ }^{+}$Applied DSP and VLSI Research Centre, Eastern Mediterranean University, Gazimagusa, Mersin 10, Turkey \\ jasi@ieee.org, M.Al-Janabi@wmin.ac.uk, kalei@wmin.ac.uk
}

Abstract- The aim of this paper is to determine the stability of higher-order $\Delta-\Sigma$ modulators using the Describing Function Method. The maximum stable input limits for third-, fourth- and fifth-order Chebyshev Type II based $\Delta-\Sigma$ modulators are established. These results are useful for optimising the design of higher-order $\Delta-\Sigma$ modulators.

\section{INTRODUCTION}

The stable input amplitude limits for $\Delta-\Sigma$ modulators is complicated to predict due to the non-linearity introduced by the quantizer in the feedback loop. Various approaches have been employed to explain this nonlinear behaviour. Using quasilinear modeling, a new interpretation of the instability mechanism for $\Delta-\Sigma$ modulators based on the noise amplification curve is given in [1]. This is restricted for dc inputs and unity quantizer gains. The quasilinear method can be extended to more than one input with each input represented by a separate equivalent gain. This concept forms the basis for the Describing Function (DF) method [2]. In this paper, the stability analysis based on the noise amplification curve is accomplished using the DF method for dc and sinusoidal inputs for non-unity quantizer gain values. In Section II, the quasilinear stability of $\Delta-\Sigma$ modulators is explained based on the noise amplification curve. In Section III, the derivation of the noise amplification curves for dc and sinusoidal inputs with the DF method is shown. The simulation results are illustrated and discussed in Section IV concluding with Section V.

\section{QUASILINEAR STABILITY ANALYSIS OF $\Delta-\Sigma$ MODULATORS}

A generic $\Delta-\Sigma$ modulator having its quantizer replaced by a gain factor $K$ followed by additive quantization noise $q(k)[1]$ is shown in Figure 1.

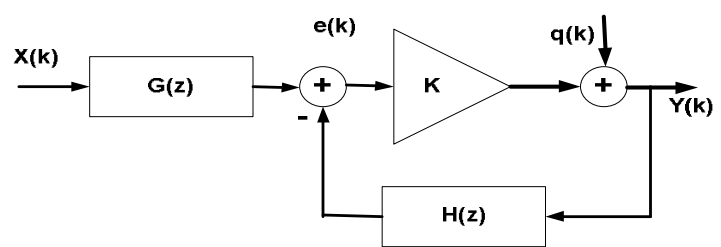

Figure 1. Quasilinear $\Delta-\Sigma$ modulator Quantizer Model.
The output of the modulator in the z-domain is given by

$$
Y(z)=\operatorname{STF}(z) X(z)+\operatorname{NTF}(z) Q(z)
$$

where, $Y(z), X(z)$ and $Q(z)$ are the z-transforms of the output, input and quantizer noise signals respectively. Also, $\operatorname{STF}(z)$ and $\operatorname{NTF}(z)$ are the Signal and Noise Transfer functions of the $\Delta-\Sigma$ modulator derived from Figure 1.

$$
\begin{aligned}
& \operatorname{STF}(z)=\frac{K \cdot G(z)}{1+K \cdot H(z)} \\
& \operatorname{NTF}(z)=\frac{1}{1+K \cdot H(z)}
\end{aligned}
$$

Equations (2) and (3) show that the poles of the denominator $(1+K H(z))$ determine the stability of the modulator. For a given $H(z)$, there will be a certain interval $\left[K_{\min }, K_{\max }\right]$ for which the modulator is stable [3]. Assuming $q(k)$ to be Gaussian white stochastic $G\left(0, \sigma_{\mathrm{q}}{ }^{2}\right)$ and the transfer function between $q(k)$ and $y(k)$ to be known, then the output noise variance is given by:

$$
\operatorname{Var}\{y(k)\}=\sigma_{q} \int_{0}^{1}\left|\operatorname{NTF}\left(e^{j \pi f}\right)\right|^{2} d f=\sigma_{q}^{2} A(K) .
$$

where, $\sigma_{\mathrm{q}}{ }^{2}$ is the variance of $q(k)$ and $A(K)$ is the total output noise power amplification factor. Using Parseval's relation, $A(K)$ can be found in the time domain as [1]

$$
A(K)=\sum_{k=0}^{\infty}|n t f(k)|^{2} \Delta\|n t f\|_{2}^{2} .
$$

where $n t f(k)$ is the impulse response corresponding to $\operatorname{NTF}(z)$ and $A(k)$ is the squared two-norm of $\operatorname{NTF}(\mathrm{z})$. The $A(K)$ curves of the loop-filter are crucial for the stability analysis of the $\Delta$ $\Sigma$ modulators. Typical curves for Type II Chebyshev $3^{\text {rd }}$ and $4^{\text {th }}$ order are shown in Figure 2. Chebyshev filters achieve better in-band Signal-to-Noise Ratio and Dynamic Range compared with Butterworth filters of the same order.

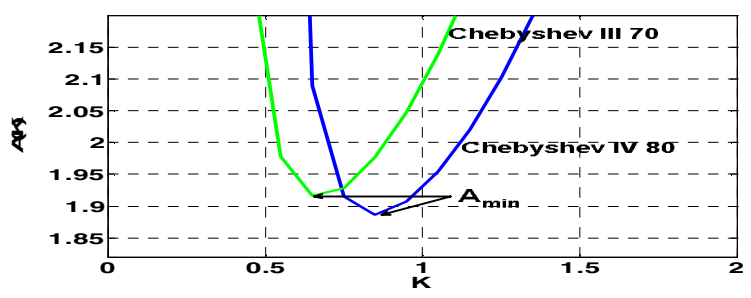

Figure 2. $A(K)$ Curves for Type II Chebyshev NTF. 
The $A_{\min }$ value is the global minimum of the curve. If $K$ increases slightly in the region where $A(K)$ is monotonically increasing, it results in a higher $A(K)$ value which leads to more quantisation noise transfer into the $\Delta-\Sigma$ modulator. This tends to decrease $K$ leading to a stable equilibrium state [1]. However, where the $A(K)$ curve is monotonically decreasing, even small perturbations can destabilize the modulator. As the signal power increases, the values along the $A(K)$ curve decrease and approach $A_{\text {min }}$. The two values of $K$ come close together and finally merge at $\mathrm{A}_{\text {min }}$. This characterizes the onset of instability. The modulator operating region escapes to the left portion of the curve where it is characterized by low values of $K$. Therefore, for stable operation $A(k)>A_{\text {min }}$.

\section{NOISE AMPLIFICATION CURVES - DF METHOD}

The quasilinear quantizer model in Figure 1 can be extended using separate gains $K_{x}$ and $K_{n}$ for the DF model as shown in Figures 3 and 4 [4].

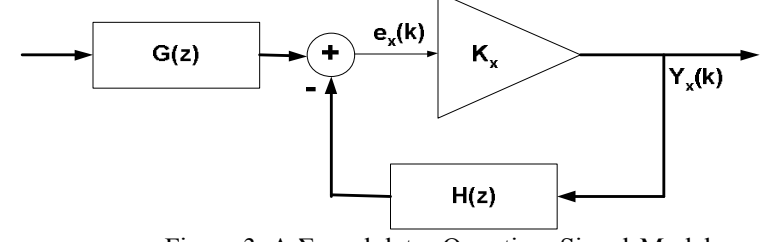

Figure 3. $\Delta-\Sigma$ modulator Quantizer Signal-Model

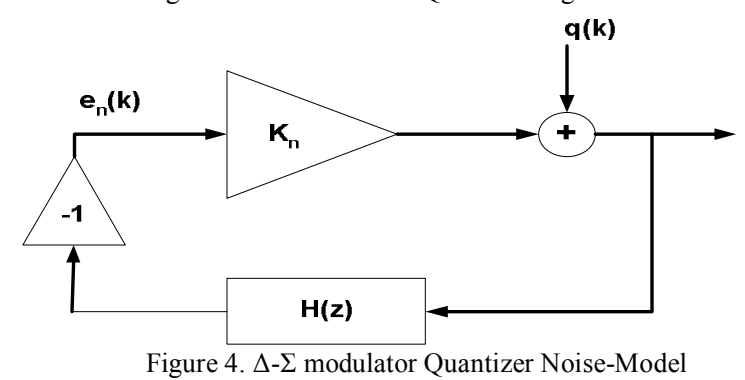

Figure 3 describes the model for the input signal with linear gain $K_{x}$ whereas Figure 4 describes the noise signal model with linear gain $K_{n}$. The combined output signal is given by:

\section{A. DC Input}

$$
y(k)=y_{x}(k)+y_{n}(k) \text {. }
$$

The linearized gains for a one bit quantizer with an output $\pm \Delta$ have been calculated in [4] as shown below:

$$
\begin{aligned}
& K_{n}=\frac{2 \Delta}{\sigma_{e_{n}}} e^{-m_{e}{ }^{2} / 2 \sigma_{e_{n}}{ }^{2} .} . \\
& K_{x}=\frac{\Delta}{m_{e}} \operatorname{erf}\left(\frac{m_{e}}{\sigma_{e_{n}} \sqrt{2}}\right) .
\end{aligned}
$$

where $m_{e}$ is the mean value of the quantizer input in the signal model and ${\sigma_{e_{n}}}^{2}$ is the noise variance input to the quantizer in the noise model. The variance of the output signal is given by:

$$
\operatorname{Var}\{y(k)\}=E\left\{y^{2}(k)\right\}-E^{2}\{y(k)\} \text {. }
$$

The output signal in the time-domain can be written as:

$$
y(k)=e_{n}(k) K_{n}+q(k)+e_{x}(k) K_{x} .
$$

The first term on the right hand side of (9) is the power of the output signal which is given by:

$$
\begin{aligned}
E\left\{y^{2}(k)\right\} & =E\left\{e_{n}{ }^{2}(k) K_{n}{ }^{2}\right\}+E\left\{q^{2}(k)\right\}+E\left\{e_{x}{ }^{2}(k) K_{x}{ }^{2}\right\} . \\
& =\sigma_{e_{n}}{ }^{2} K_{n}{ }^{2}+\sigma_{q}{ }^{2}+m_{e}{ }^{2} K_{x}{ }^{2} .
\end{aligned}
$$

As the mean values of $e_{n}(k)$ and $q(k)$ are equal to zero, then the second term on the right hand side of (9) is:

$$
E^{2}\{y(k)\}=m_{e}{ }^{2} K_{x}^{2} .
$$

The resultant variance of the output signal using (9), (12) and (13) becomes:

$$
\operatorname{Var}\{y(k)\}=\sigma_{e_{n}}{ }^{2} K_{n}{ }^{2}+\sigma_{q}{ }^{2} .
$$

The noise power amplification factor for a dc input signal $A_{d c}(K)$ after using (4), (7) and (14) simplifies to:

$$
A_{d c}(K)=\frac{\operatorname{Var}\{y(k)\}}{\sigma_{q}^{2}}=\frac{\left(\frac{2}{\pi}\right)\left[e^{-\lambda^{2}}\right]^{2}+\sigma_{q}^{2}}{\sigma_{q}^{2}} .
$$

where $\lambda$ is a factor defined as: $\lambda=m_{e} / \sigma_{e n} \sqrt{ } 2$ and $\sigma_{q}{ }^{2}$ is the quantization noise given by [4]

$$
\sigma_{q}^{2}=\Delta^{2}\left[1-\frac{m_{x}}{\Delta^{2}}-\frac{2}{\pi} e^{-2\left[\operatorname{erf}^{-1}\left(\frac{m_{x}}{\Delta}\right)\right]^{2}}\right] .
$$

\section{B. Sinusoidal Input}

The linearised gains for a sinusoidal input and random Gaussian feedback components have been solved for the case of an ideal relay in [5] and are shown below:

$$
\begin{aligned}
& K_{n}=\left(\frac{2}{\pi}\right)^{\frac{1}{2}}\left(\frac{\Delta}{\sigma_{e_{n}}}\right) F\left(\frac{1}{2}, 1,-v^{2}\right) . \\
& K_{x}=\left(\frac{2}{\pi}\right)^{\frac{1}{2}}\left(\frac{\Delta}{\sigma_{e_{n}}}\right) F\left(\frac{1}{2}, 2,-v^{2}\right) .
\end{aligned}
$$

Here, $v \Delta \frac{a}{\sqrt{2}} \frac{1}{\sigma_{e_{n}}}$, where $a$ is the amplitude of the sinusoidal input signal $x(k)$. The expression $F(\alpha, \gamma, x)$ is the confluent hypergoemetric function defined by [6]: 


$$
F(\alpha, \gamma, \chi) \underline{\underline{\Delta} 1}+\frac{\alpha \chi}{\gamma}+\frac{\alpha(\alpha+1) \chi^{2}}{\gamma(\gamma+1) \Gamma 2}+\ldots
$$

The variance of the output signal is given by:

$$
\operatorname{Var}\{y(k)\}=E\left\{y^{2}(k)\right\}-E^{2}\{y(k)\} .
$$

The power of the output signal is given by:

$$
\begin{aligned}
E\left\{y^{2}(k)\right\} & =E\left\{e_{n}{ }^{2}(k) K_{n}{ }^{2}\right\}+E\left\{q^{2}(k)\right\}+E\left\{e_{x}{ }^{2}(k) K_{x}{ }^{2}\right\} . \\
& =\sigma_{e_{n}}{ }^{2} K_{n}{ }^{2}+\sigma_{q_{s}}{ }^{2}+\sigma_{e_{x}}{ }^{2} K_{x}{ }^{2}
\end{aligned}
$$

where, $\sigma_{q s}{ }^{2}$ is the quantization noise power for a sinusoidal input. The second term on the right hand side of (20) is:

$$
\begin{aligned}
E^{2}\{y(k)\} & =E^{2}\left\{e_{n}(k) K_{n}\right\}+E^{2}\{q(k)\}+E^{2}\left\{e_{x}(k) K_{x}\right\}(23) \\
& =E^{2}\left\{e_{x}(k)\right\} K_{x}{ }^{2}
\end{aligned}
$$

where the mean values of $e_{n}(k)$ and $q(k)$ are zero. Since the input signal is a sinusoid modelled as a Random Variable (RV) with a certain amplitude and phase having a uniform Probability Density Function (PDF). Therefore, $E\left\{e_{x}(k)\right\}=0$.

$$
\operatorname{Var}\{y(k)\}=\sigma_{q_{s}}{ }^{2}+K_{n}{ }^{2} \sigma_{e_{n}}{ }^{2}+\sigma_{e_{x}}{ }^{2} K_{x}{ }^{2} .
$$

Given that the frequency of $x(k)$ is small in the baseband region, this then results in:

$$
\frac{E_{x}(z)}{X(z)} \approx \frac{1}{K_{x}} .
$$

The variance of $e_{x}(k)$ is:

$$
\sigma_{e_{x}}^{2}=\frac{1}{K_{x}^{2}} \sigma_{x}^{2}
$$

From (25) and (27), the output signal variance is:

$$
\operatorname{Var}\{y(k)\}=\sigma_{q_{s}}{ }^{2}+K_{n}{ }^{2} \sigma_{e_{n}}{ }^{2}+\sigma_{x}^{2} .
$$

The output noise variance is therefore:

$$
\operatorname{Var}\{y(k)\}=\sigma_{q_{s}}{ }^{2}+K_{n}{ }^{2}{\sigma_{e_{n}}}^{2} .
$$

Substituting (17) in (29), the noise amplification factor for a sinusoidal input signal is given by:

$$
A_{\sin e}(K)=\frac{\left(\frac{2}{\pi}\right) F^{2}\left(\frac{1}{2}, 1,-v^{2}\right)+\sigma_{q_{S}}{ }^{2}}{\sigma_{q_{S}}{ }^{2}} .
$$

The values of $v$ and $\sigma_{q s}{ }^{2}$ can be found using the following expressions derived in [4]:

$$
\begin{aligned}
& v^{2} F^{2}\left(\frac{1}{2}, 2,-v^{2}\right)=\frac{\pi}{4}\left(\frac{a^{2}}{\Delta^{2}}\right) . \\
& \sigma_{q_{S}}{ }^{2}=\Delta^{2}\left[1-\frac{a^{2}}{2 \Delta^{2}}-\frac{2}{\pi} F^{2}\left(\frac{1}{2}, 1,-v^{2}\right)\right] .
\end{aligned}
$$

\section{RESULTS \& SIMULATIONS}

The variation of the dc and sinusoidal input quantisation noise $\sigma_{q}^{2}$ and $\sigma_{s}^{2}$ with respect to the input signal amplitude using (16) and (32) are shown in Figure 5. As can be seen, $\sigma_{\mathrm{q}}{ }^{2}$ decreases and becomes zero as the input signal amplitude increases to unity. The quantization noise $\sigma_{s}^{2}$ does not decrease to zero and remains at 0.3 for an input amplitude of 1.0 .

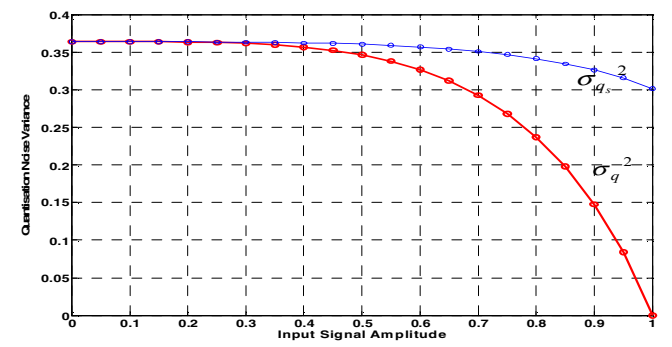

Figure 5. Quantization Noise for dc \& Sinusoidal Inputs

Equation (31) has been solved for $v$ up to the $10^{\text {th }}$ power of the polynomial using a MATLAB routine. Figure 6 shows the variation of $\lambda$ and $v$ with respect to the input signal amplitude.

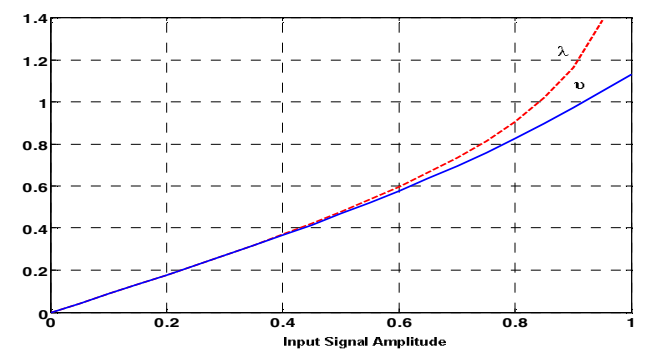

Figure 6. Variation of $v \& \lambda$.

It has been observed that for amplitudes less than 0.4, the quantization noise, $\lambda$ and $v$ are almost the same for dc and sinusoidal inputs. This coincides with the fact that in nonlinear feedback systems, the effective gain of the non-linearity on a small signal is independent of the type of signal [2]. The noise amplification factors $A_{d c}(K)$ and $A_{\sin }(K)$ using (15) and (30) are illustrated in Figure 7. It is seen that the values of $A_{d c}(k)$ using the DF method are the same as in [1].

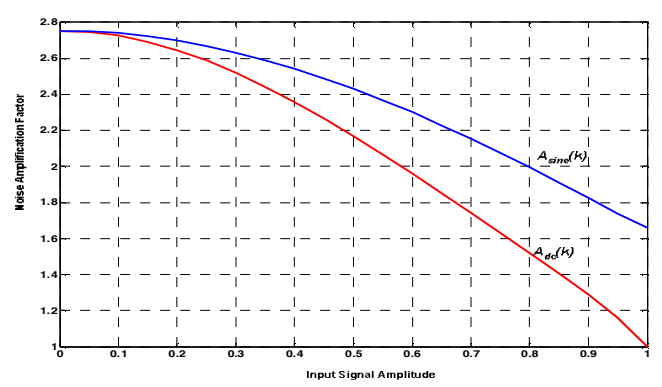

Figure 7. Noise Amplification Factor for sinusoidal \& dc inputs 
Using $A_{d c}(k)$ and $A_{\text {sine }}(k)$, the maximum stable input amplitudes for the $3^{\text {rd }}, 4^{\text {th }}$ and $5^{\text {th }}$ order Chebyshev Type II based $\Delta-\Sigma$ modulator are demonstrated in Figure 8.

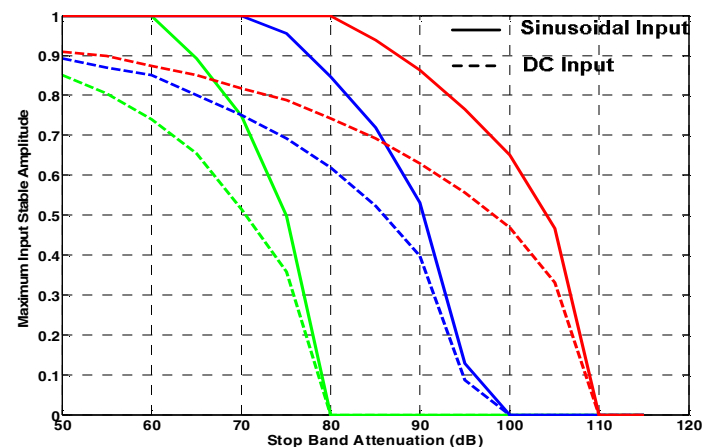

Figure 8. Maximum stable input amplitude for Chebyshev Type II NTF(z).

However, these are true for unity values of $K$. The variation of the stable sinusoidal input amplitude for a $4^{\text {th }}$-order Chebyshev Type II based $\Delta-\Sigma$ modulator in relation to $K$ and the stopband attenuation is shown in Figure 9.

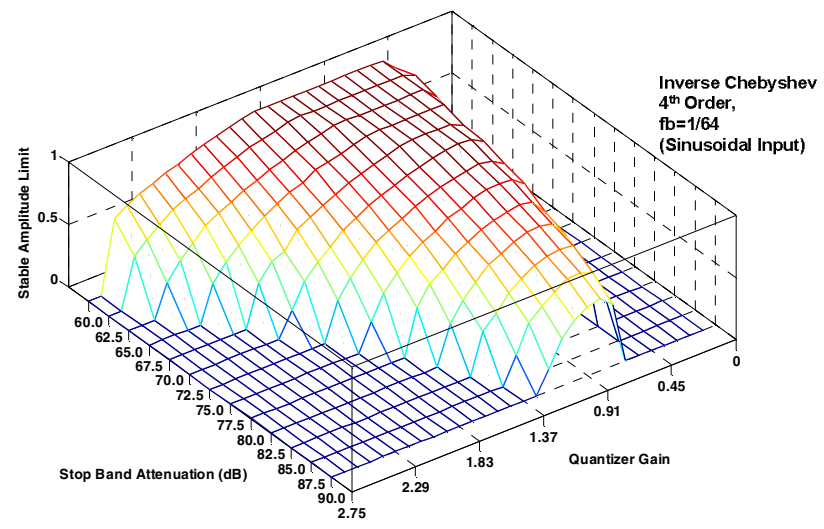

Figure 9. Stable amplitude variation with Quantizer Gain \& stop band.

The stable input amplitude variation for $\mathrm{dc}$ and sinusoidal inputs to a $5^{\text {th }}$ order Chebyshev Type II based $\Delta-\Sigma$ modulator (stop band attenuation at $67 \mathrm{~dB}$ ) is shown in Figure 10.

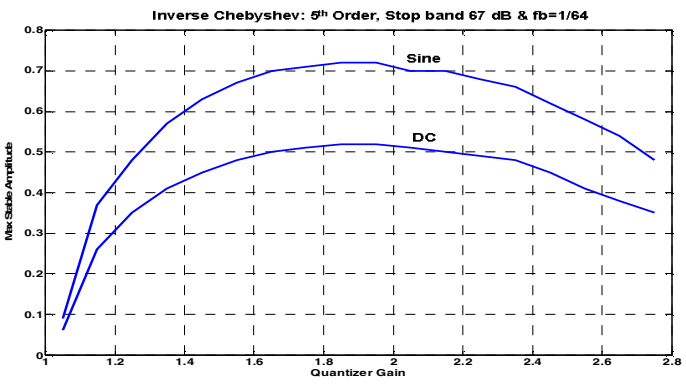

Figure 10. Stable amplitude variation with Quantizer Gain
Simulations for the $5^{\text {th }}$-order Chebyshev Type II based $\Delta-\Sigma$ modulator implemented in the feed forward topology were undertaken for 1638400 time samples with an increase in input amplitude in steps of 0.1 . The maximum stable amplitude limits were observed and the corresponding values of $K$ were calculated using (33) [7]

$$
K=\text { Covariance }\{e(k), y(k)\} / \sigma_{e}^{2}
$$

The predicted values of the maximum stable amplitudes were obtained from Figure 10.

Table I. SIMULATION RESULTS.

\begin{tabular}{|c|c|c|c|}
\hline Signal & $\boldsymbol{K}$ & $\begin{array}{c}\text { Predicted Stable } \\
\text { Amplitude }\end{array}$ & $\begin{array}{c}\text { Stable Amplitude } \\
\text { As per Simulations }\end{array}$ \\
\hline $\mathbf{d c}$ & 1.62 & 0.52 & 0.63 \\
\hline sine & 1.70 & 0.69 & 0.66 \\
\hline
\end{tabular}

The difference in values is attributed to the composition of the quantization noise which is not entirely Gaussian [7].

\section{CONCLUSION}

The stability of higher-order $\Delta-\Sigma$ modulators for $\mathrm{dc}$ and sinusoidal inputs using the Describing Function Method has been predicted. The maximum stable input limits for the $3^{\text {rd }}$-, $4^{\text {th }}$ - and $5^{\text {th }}$-order Chebyshev Type II based $\Delta-\Sigma$ modulator have been established for a unity quantizer gain. More accurate results for the stable amplitude curves can be obtained for a range of values of quantizer gain $K$ in which the $\Delta-\Sigma$ modulators are likely to operate. A future publication would include the analysis for multiple sinusoidal inputs.

\section{REFERENCES}

[1] Risbo, L., "Stability Predictions for Higher-Order Sigma-Delta Modulators Based on Quasilinear Modeling", IEEE International Symposium on Circuits \& Systems, Volume 5, page 361-364, 1994.

[2] Gelb, A., Vander Velde, W., E., Multiple-Input Describing Functions and Nonlinear System Design, New York McGraw-Hill, 1968.

[3] Stikvoort, E., F., "Some Remarks on the Stability and Performance of the Noise Shaper or Sigma-Delta Modulator" IEEE Trans. On Communications, Volume 36, no.10, page 1157-1162, Oct 1988.

[4] Ardalan, S., H., Paulos, J., "An Analysis of Nonlinear Behavior in DeltaSigma Modulators", IEEE Transactions on Circuits \& Systems, Volume CAS34, No.6, June 1987.

[5] Atherton, D., P., Nonlinear Control Engineering-Describing Function Analysis \& Design, Van Notsrand Reinhold London, page 383-388, 1982.

[6] Haddad, A., H., Nonlinear Systems, Benchmark Papers in Electrical Engineering and Computer Science, vol. 10, Dowden, Hutchinson \& Ross, Inc and Halsted Press, page 197, 1975.

[7] Risbo, L., Sigma-Delta Modulators- Stability Analysis and Optimisation, PhD Thesis, Electronics Institute, Technical University of Denmark, June 1994. 